\title{
Assessment of ErbB2 (Her2) in oesophageal adenocarcinomas: summary of a revised immunohistochemical evaluation system, bright field double in situ hybridisation and fluorescence in situ hybridisation
}

\author{
Rupert Langer ${ }^{1}$, Sandra Rauser ${ }^{2}$, Marcus Feith ${ }^{3}$, Jörg M Nährig ${ }^{1}$, Annette Feuchtinger ${ }^{2}$, \\ Helmut Friess ${ }^{3}$, Heinz Höfler ${ }^{1,2}$ and Axel Walch ${ }^{2}$ \\ ${ }^{1}$ Institute of Pathology, Technische Universität München, Munich, Germany; ${ }^{2}$ Institute of Pathology, \\ Helmholtz Zentrum München, German Research Center for Environmental Health, Neuherberg, Germany \\ and ${ }^{3}$ Department of Surgery, Klinikum rechts der Isar, Technische Universität München, Munich, Germany
}

\begin{abstract}
Amplification and overexpression of ErbB2 (Her2) is a frequent event in oesophageal adenocarcinomas. Assessment of ErbB2 status is crucial for identifying patients who are likely to benefit from treatment with trastuzumab. In this study, we performed a comprehensive analysis of ErbB2 amplification and expression in 142 oesophageal adenocarcinomas by comparing the most commonly used methods for ErbB2 assessment: ErbB2 expression was determined by immunohistochemistry and was scored $(0,1+, 2+$ and $3+)$ according to a recently described modified scoring system for gastric cancer. ErbB2 amplification was evaluated by bright field double in situ hybridisation. The results were compared with pathologic features, patients' survival and previously published data from fluorescence in situ hybridisation analysis. On the basis of immunohistochemistry, which was applicable in 110 cores of the cases, 83 tumours (75\%) had a score of 0 or $1+$ (immunohistochemistry negative), 13 tumours (12\%) were scored as $2+$ and 14 tumours $(13 \%)$ were scored as $3+$. In situ hybridisation data were obtained from 142 cases. There was a highly significant correlation of immunohistochemistry, bright field in situ hybridisation and fluorescent in situ hybridisation $(\boldsymbol{P}<0.001$ each). In total, 41 tumours $(29 \%)$ were categorised as ErbB2 positive, which was defined as immunohistochemistry $3+$ and/or an ErbB2/Chr17 quotient of $\geq 2$ as assessed by either bright field double in situ hybridisation or fluorescence in situ hybridisation. ErbB2 positivity was observed more frequently in tumours with lower differentiation grades $(P=0.029)$. Patients with ErbB2-positive tumours had a significantly worse prognosis, both in univariate analysis $(P=0.004)$ and in multivariate analysis $(P=0.03)$. In conclusion, we demonstrate that a significant number of oesophageal adenocarcinomas are positive for ErbB2. Assessment of ErbB2 amplification can be equivalently performed by conventional fluorescence in situ hybridisation or other lightmicroscopy-based methods, such as the novel bright field double in situ hybridisation technique. Modern Pathology (2011) 24, 908-916; doi:10.1038/modpathol.2011.52; published online 22 April 2011
\end{abstract}

Keywords: adenocarcinoma; ErbB2; immunohistochemistry; in situ hybridisation; oesophagus

ErbB2 (Her2) amplification or overexpression occurs frequently in adenocarcinomas of the upper gastro-

Correspondence: Dr R Langer, MD, Institute of Pathology, Klinikum Rechts der Isar, Technische Universität München, Trogerstrasse 18, München D-81675, Germany.

E-mail: rupert.langer@lrz.tu-muenchen.de

Received 13 December 2010; revised 11 February 2011; accepted 16 February 2011; published online 22 April 2011 intestinal tract. Most studies have been conducted in gastric cancer, which shows ErbB2-positivity rates that vary from 10 to $30 \% \cdot{ }^{1-5}$ ErbB2 amplification or overexpression also seems to be associated with poorer prognosis, more aggressive disease and shorter survival in gastric cancer. ${ }^{5-7}$ Preclinical and clinical studies of upper gastrointestinal adenocarcinomas, ${ }^{2}$ and in particular, the large ToGA trial, which included adenocarcinomas of the stomach 
and gastro-oesophageal junction, have demonstrated that trastuzumab exerts antitumour activity on ErbB2-overexpressing tumours. ${ }^{8}$ Most recently, this has led to the approval of the addition of trastuzumab to fluoropyrimidine/platinum-based therapies for patients with ErbB2-positive advanced gastric cancer by the European Medicines Agency (EMEA), the FDA and Health Canada.

Similar to breast cancer, ${ }^{9}$ determination of ErbB2 status is performed by immunohistochemistry as the primary method of choice. In situ hybridisation, which is mostly performed as fluorescence in situ hybridisation, is restricted to those cases that have equivocal (ie, immunohistochemistry 2+) ErbB2 expression results. There is evidence that compared to breast cancer, ErbB2 immunohistochemistry in gastrointestinal carcinomas has a few substantial differences; in particular, these differences involve cellular staining patterns and intratumoral heterogeneity. Therefore, for gastric cancer, a modified ErbB2 scoring system for immunohistochemistry has been proposed. ${ }^{3,10}$

Data on ErbB2 in oesophageal adenocarcinomas exist to a lesser extent than gastric cancer. The rate of ErbB2 overexpression/amplification in these cancers has been reported at approximately $20-25 \%$, depending on the method of evaluation and the definition used. ${ }^{11-15}$ In terms of the relatively high rate of chemotherapy resistance in oesophageal adenocarcinomas, ${ }^{16,17}$ additional therapeutic options, such as ErbB2-targeting are of high demand for patients with advanced or metastatic disease.

The aim of this study was to perform a concise analysis of ErbB2 expression in a well-characterised series of oesophageal adenocarcinomas. For this purpose, we applied the revised ErbB2 immunohistochemistry scoring recommendations for gastric cancer $^{3,10}$ to a tissue microarray that comprised a large number of primary resected oesophageal adenocarcinomas. In addition, in situ hybridisation was performed using the recently developed bright field double in situ hybridisation. ${ }^{18}$ Results were compared with fluorescence in situ hybridisation data from a previously published study by our group to assess the reliability of both the modified scoring system for immunohistochemistry and the novel in situ hybridisation technique. Furthermore, the expression and amplification data were correlated with histopathological parameters and patients' survival.

\section{Materials and methods}

\section{Patients}

Immunohistochemistry and in situ hybridisation were performed on paraffin-embedded formalinfixed tumour samples from a total of 142 patients with oesophageal adenocarcinomas who were treated between 1991 and 2006 in the Department of
Surgery at the Klinikum Rechts der Isar der Technischen Universität München. All patients provided consent for additional molecular analyses at the time of their original operation. Mean patient age was 64 years, and ages ranged from 33 to 83 years. The female/male ratio was 12/130. Median disease-free survival was 44 months (95\% CI; 29-58 months). Median overall survival was 49 months (95\% CI; 31-67 months). All patients had been treated by radical surgical resection-either transthoracic or transhiatal oesophagectomy-without neoadjuvant chemotherapy or radiochemotherapy. The pT category of the tumours (according to UICC $2010^{19}$ ) was as follows: pT1, 63 cases (44\%); pT2, 28 cases (20\%); and pT3-pT, 4-51 cases (36\%). Lymph node metastases were present in 59 cases $(42 \%)$. Tumour grading was G1 (well differentiated) in 12 cases (9\%), G2 (moderately differentiated) in 62 cases (44\%) and G3-G4 (poorly differentiated) in 68 cases $(48 \%$; Table 1$)$.

\section{Tissue Microarrays}

Tissue microarrays constructed at two different time points were used. Fluorescence in situ hybridisation analysis was done on the first tissue microarray, which contained cores from 124 tumours ${ }^{11,12}$ with three cores per tumour. Results of this study were originally published in 2006 and 2007. For the purpose of this study, a second tissue microarray was constructed, which contained samples of 110 with two cores per tumour. Overall, 94 tumours of

Table 1 ErbB2 (Her2) status in oesophageal adenocarciomas and pathological parameters

\begin{tabular}{lrrrr}
\hline & \multicolumn{2}{c}{ ErbB2 status } & Total & $\begin{array}{c}\text { P-value } \\
\left(\chi^{2}\right)\end{array}$ \\
\cline { 2 - 3 } & Negative & Positive & & \\
\cline { 2 - 3 }$p$ Category & & & & \\
pT1 & 50 & 13 & 63 & 0.154 \\
pT2 & 18 & 10 & 28 & \\
pT3-4 & 33 & 18 & 51 & \\
Total & 101 & 41 & 142 & \\
& & & & \\
Lymph node metastases & & & & \\
Absent & 64 & 19 & 83 & 0.090 \\
Present & 37 & 22 & 59 & \\
Total & 101 & 41 & 142 & \\
& & & & \\
Distant metastases & & & & \\
Absent & 94 & 38 & 132 & 1.00 \\
Present & 7 & 3 & 10 & \\
Total & 101 & 41 & 142 & \\
Grading & & & & \\
G1 & & & & \\
G2 & 12 & 0 & 12 & 0.029 \\
G3-4 & 39 & 23 & 62 & \\
Total & 50 & 18 & 68 & \\
& 101 & 41 & 142 & \\
\hline
\end{tabular}

ErbB2 positivity is defined as immunohistochemistry $3+$ and/or an ErbB2/Chr17 quotient of $\geqslant 2$ as assessed by in situ hybridisation. 
the first tissue microarray could be included in replicate, whereas 30 tumours could not be included in the second tissue microarray due to the decreased tumour content in the donor paraffin blocks, which particularly occurred with the early carcinomas. Instead, 16 other tumours were added to the second tissue microarray.

\section{Immunohistochemistry}

The paraffin blocks of the tissue microarrays were freshly cut into $3 \mu \mathrm{m}$ sections that were incubated with antibodies against ErbB2 (clone 4B5, Ventana Medical Systems Inc., Tucson, Arizona) according to the manufacturer's recommendations. The method of ErbB2 scoring was essentially based on previously described recommendations, which was also applied in the ToGA study for gastric cancer and is described in detail elsewhere ${ }^{3,10}$ Briefly, the major features of the modification that are different from the ErbB2 scoring in breast cancer are as follows: a positive reaction is considered a membranous staining pattern that can be unequivocally assessed as linear staining at cell-cell contact sites and includes baso-lateral or lateral without restriction on the circularity of staining; the degree of microscopic magnification (x-fold) at which the membranous (linear intercellular) staining is clearly visible for the estimation of ErbB2 staining intensity is considered; the $10 \%$ minimum number of stained tumour cells that could reliably be assessed for ErbB2 staining in biopsies is eliminated. In this study, at least five cohesive, unequivocally stained evaluable cells are considered to be the limit for ErbB2 evaluation (Figure 1). In this study, we followed this recommendation because the tumour content of a tissue microarray core may actually represent that of a biopsy rather than a slice of a resection specimen.

\section{In Situ Hybridisation}

Bright field in situ hybridisation was performed using the BenchMark ${ }^{\mathbb{R}}$ XT automated slide processing system (Ventana Medical Systems Inc.), which involves a one-step fully automated assay that consists of baking, deparaffinisation, pretreatment, hybridisation, stringency wash, signal detection and counterstaining as described in detail in a recent publication. ${ }^{18}$ For ErbB2 gene detection, the Inform ${ }^{\circledR}$ ErbB2 DNA Probe (Ventana Medical Systems Inc.) was used, and for Chr 17 detection, the Inform Chromosome 17 Probe (Ventana Medical Systems Inc.) was used (Figure 1).

Additional data from conventional fluorescence in situ hybridisation using $4 \mu \mathrm{m}$ tissue microarray sections and three-dimensional (3D) fluorescence in situ hybridisation using $16 \mu \mathrm{m}$ tissue microarray sections were obtained from a previously published study by our group. ${ }^{12}$
For the purpose of this study, cases with an ErbB2/Chr17 ratio of $\geq 2$ based on either conventional or 3D fluorescence in situ hybridisation were classified as ErbB2 amplified. ErbB2 amplification was further subclassified into low-level amplification (ErbB2/Chr17 ratio 2-3) and high-level amplification (ErbB2/Chr17 ratio >3), consistent with published studies. ${ }^{13}$

\section{Statistical Analysis}

PASW 18.0 statistical software 11.5 (SPSS Inc., Chicago, IL) was used for statistical analysis. The associations between immunohistochemical expression patterns, the results from in situ hybridisation and the pathologic features were entered into $2 \times 2$ tables and evaluated with the $\chi^{2}$-test. Survival analysis was performed using Kaplan-Meier estimates, log-rank tests and Cox's proportional hazards regression analysis. All tests were two-sided, and the significance level was set at $5 \%$.

\section{Results}

\section{Immunohistochemistry}

On the basis of the immunohistochemistry, which was applicable in 110 cores of the tissue microarray, 83 tumours $(75 \%)$ had a score of 0 or $1+$ (immunohistochemistry negative), 13 tumours $(12 \%)$ were scored as $2+$ and 14 tumours (13\%) were scored as $3+$. In two cases, there was an obvious intratumoral heterogeneity with divergent staining patterns (negative vs $2+$ or $3+$ ) in adjacent tumour areas. We also had the opportunity to compare the breast cancer scoring system, which we used for the ErbB2 assessment in oesophageal adenocarcinomas in a previous study, with the proposed scoring system for gastric cancer: 94 tumours were contained in both tissue microarrays, and significant $(P<0.001)$ concordant scoring that ranged from negative to $3+$ was observed in 56 cases $(60 \%)$, or in 77 cases $(82 \%)$, if negative and $1+$ cases were grouped together (Table 2).

\section{In Situ Hybridisation}

In situ hybridisation data were obtained from a total of 142 tumours on tissue microarrays as indicated above. Bright field double in situ hybridisation showed a low-level amplification of ErbB2 (ErbB2/ Chr17 ratio 2-3) in 11 cases (10\%) and a high-level amplification (ErbB2/Chr17 ratio $>3$ ) in 15 cases (14\%). No amplification of ErbB2 (ErbB2/Chr17 ratio $<2)$ was observed in 81 cases $(76 \%)$. There was a significant correlation between bright field double in situ hybridisation data, both with conventional fluorescence in situ hybridisation and 3D fluorescence in situ hybridisation $(P<0.001$ each). In three cases, there was an intratumoral heterogeneity 

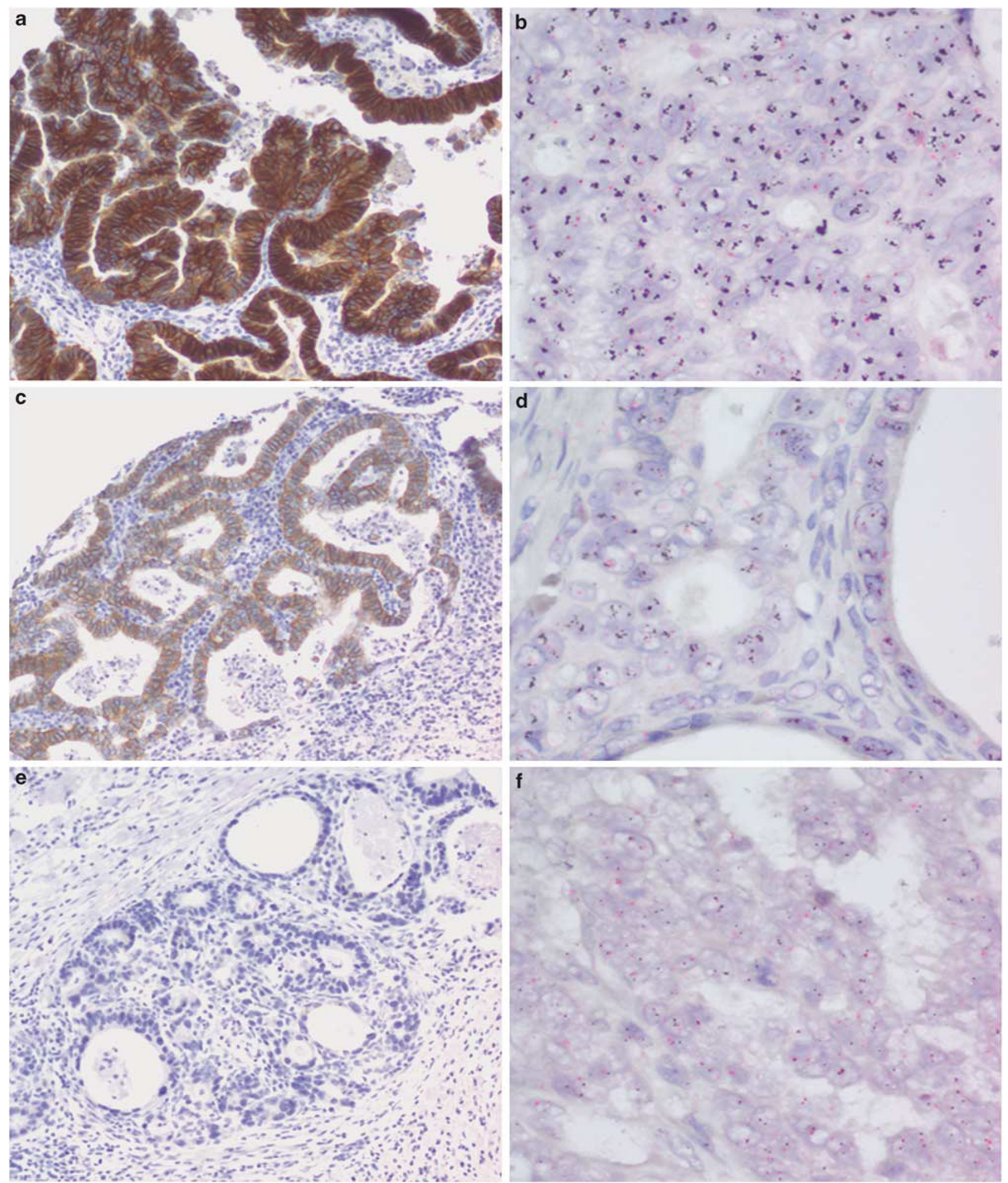

Figure 1 Examples of immunohistochemical stainings and bright field in situ hybridisation for ErbB2 (Her2) in oesophageal adenocarcinomas. (a) ErbB2 immunohistochemistry score 3+ (×200); (b) same case, ErbB2 high-level amplification with clustering of ErbB2 signals. ErbB2 signals are black; Chr17 signals are red ( $\times 400)$. (c) ErbB2 immunohistochemistry score $2+(\times 200)$; (d) same case ErbB2 low-level amplification with a ratio of ErbB2/Chr17 $2.6(\times 400)$. (e) ErbB2 immunohistochemistry score $0(\times 200)$; $(\mathbf{f})$ same case, ErbB2 non-amplified $(\times 400)$.

(no amplification vs high-level amplification in adjacent tumour areas) within the tissue microarray cores. This was congruent to immunohistochemical findings in two of these cases as described above. In addition, there was discordance in the evaluation of high-level amplification (present vs absent) in five 
cases (5.6\%) for the comparison between bright field double in situ hybridisation and fluorescence in situ hybridisation and in six cases $(7 \%)$ for bright field in situ hybridisation and 3D fluorescence in situ hybridisation (Table 3). However, this finding may also be caused by intratumoral heterogeneity as discussed below.

\section{Correlation between Immunohistochemistry and In Situ Hybridisation}

There was a highly significant correlation between immunohistochemistry and in situ hybridisation

Table 2 Comparison between the ErbB2 (Her2) gastric cancer grading system and the breast cancer grading system

\begin{tabular}{|c|c|c|c|c|c|}
\hline & \multicolumn{4}{|c|}{ ErbB2 IHC gastric cancer grading } & \multirow[t]{2}{*}{ Total } \\
\hline & 0 & $1+$ & $2+$ & $3+$ & \\
\hline \multicolumn{6}{|c|}{ ErbB2 IHC breast cancer grading } \\
\hline 0 & 42 & 16 & 3 & 3 & 64 \\
\hline $1+$ & 5 & 3 & 4 & 0 & 12 \\
\hline $2+$ & 3 & 1 & 3 & 1 & 8 \\
\hline $3+$ & 1 & 0 & 1 & 8 & 10 \\
\hline Total & 51 & 20 & 11 & 12 & 94 \\
\hline
\end{tabular}

IHC, immunohistochemistry.

Significant correlation between both grading systems $\left(\chi^{2}\right.$-test; $\left.P<0.001\right)$.

Table 3 Comparison between for bright field double in situ hybridisation, fluorescence in situ hybridisation and 3D fluorescence in situ hybridisation

\begin{tabular}{|c|c|c|c|c|c|c|c|c|}
\hline & \multicolumn{3}{|c|}{$\begin{array}{c}\text { FISH } \\
\text { (ErbB2/Chr17) }\end{array}$} & \multirow[t]{2}{*}{ Total } & \multicolumn{3}{|c|}{$\begin{array}{c}3 D-F I S H \\
\text { (ErbB2/Chr17) }\end{array}$} & \multirow[t]{2}{*}{ Total } \\
\hline & $<2$ & $2-3$ & $>3$ & & $<2$ & $2-3$ & $>3$ & \\
\hline \multicolumn{9}{|c|}{ BDISH (ErbB2/Chr17) } \\
\hline$<2$ & 64 & 3 & 1 & 68 & 62 & 7 & 1 & 70 \\
\hline $2-3$ & 8 & 1 & 1 & 10 & 6 & 2 & 2 & 10 \\
\hline$>3$ & 4 & 0 & 8 & 12 & 5 & 0 & 7 & 12 \\
\hline Total & 76 & 4 & 10 & 90 & 73 & 9 & 10 & 92 \\
\hline
\end{tabular}

BDISH, bright field double in situ hybridisation; FISH, fluorescence in situ hybridisation.

Significant correlation between the results of BDISH and FISH $\left(P<0.001 ; \chi^{2}\right.$-test $)$ and BDISH and 3D-FISH $\left(P<0.001 ; \chi^{2}\right.$-test $)$.
$(P<0.001$; for bright field double in situ hybridisation, fluorescence in situ hybridisation, 3D fluorescence in situ hybridisation and in situ hybridisation in total): 12/13 ErbB2 $2+$ cases and 13/13 ErbB2 $3+$ cases were amplified with in situ hybridisation. However, 10 cases with a ErbB2 immunohistochemical score of 0 and 3 cases with a ErbB2 score of $1+$ were also amplified, showing low-level amplification in the majority of cases (11/13 cases). Four ErbB2 $3+$ cases were not amplified by fluorescence in situ hybridisation analysis. However, bright field double in situ hybridisation detected amplification in all of those cases, indicating intratumoral heterogeneity of ErbB2. In contrast, 12 cases that were negative by bright field double in situ hybridisation showed amplification with either conventional fluorescence in situ hybridisation or 3D fluorescence in situ hybridisation. In addition, one ErbB2 3 + case that had been added to the second tissue microarray and had not been included in the fluorescence in situ hybridisation collective could not be analysed by bright field double in situ hybridisation due to technical reasons (Table 4).

\section{Correlation between ErbB2 Status and Pathological Parameters}

In total, 41 tumours (29\%) were categorised as ErbB2 positive, which was defined as $3+$ by immunohistochemistry and/or a ErbB2/Chr17 quotient of $\geq 2$ as assessed by either bright field double in situ hybridisation or fluorescence in situ hybridisation. ErbB2 positivity was observed more frequently in tumours with a lower differentiation grade $(P=0.029)$. There was no correlation between ErbB2 status and pT and pN category (Table 1).

\section{ErbB2 Status and Survival}

Patients with ErbB2-positive tumours had a significantly worse clinical outcome, with a significantly shorter disease-free survival and a significantly decreased overall survival: the median disease-free survival for patients with ErbB2-negative tumours was 60 months (95\% CI; 22-97 months), and the

Table 4 Comparison between ErbB2 immunohistochemistry and in situ hybridisation

\begin{tabular}{|c|c|c|c|c|c|c|c|c|c|c|c|c|}
\hline & \multirow[t]{2}{*}{ Total } & \multicolumn{2}{|c|}{ ISH total } & \multicolumn{3}{|c|}{ FISH (ErbB2/Chr17) } & \multicolumn{3}{|c|}{ 3D FISH (ErbB2/Chr17) } & \multicolumn{3}{|c|}{ B-DISH (ErbB2/Chr17) } \\
\hline & & Not amplified & Amplified & $<2$ & $2-3$ & $>3$ & $<2$ & $2-3$ & $>3$ & $<2$ & $2-3$ & $>3$ \\
\hline \multicolumn{13}{|l|}{$I H C$} \\
\hline Negative & 58 & 48 & 10 & 47 & 1 & 1 & 46 & 4 & 1 & 56 & 2 & 0 \\
\hline $1+$ & 25 & 22 & 3 & 18 & 1 & 0 & 17 & 3 & 0 & 23 & 0 & 0 \\
\hline $2+$ & 13 & 1 & 12 & 7 & 2 & 1 & 8 & 1 & 2 & 2 & 6 & 5 \\
\hline $3+$ & 14 & 1 & 13 & 4 & 0 & 8 & 4 & 1 & 7 & 0 & 3 & 10 \\
\hline Total & 110 & 72 & 38 & 76 & 4 & 10 & 75 & 9 & 10 & 81 & 11 & 15 \\
\hline
\end{tabular}

BDISH, bright field double in situ hybridisation; FISH, fluorescence in situ hybridisation; ISH, in situ hybridisation.

Significant correlation between IHC and FISH, 3D-FISH and BDISH ( $\chi^{2}$-testing; $P<0.001$ each). 

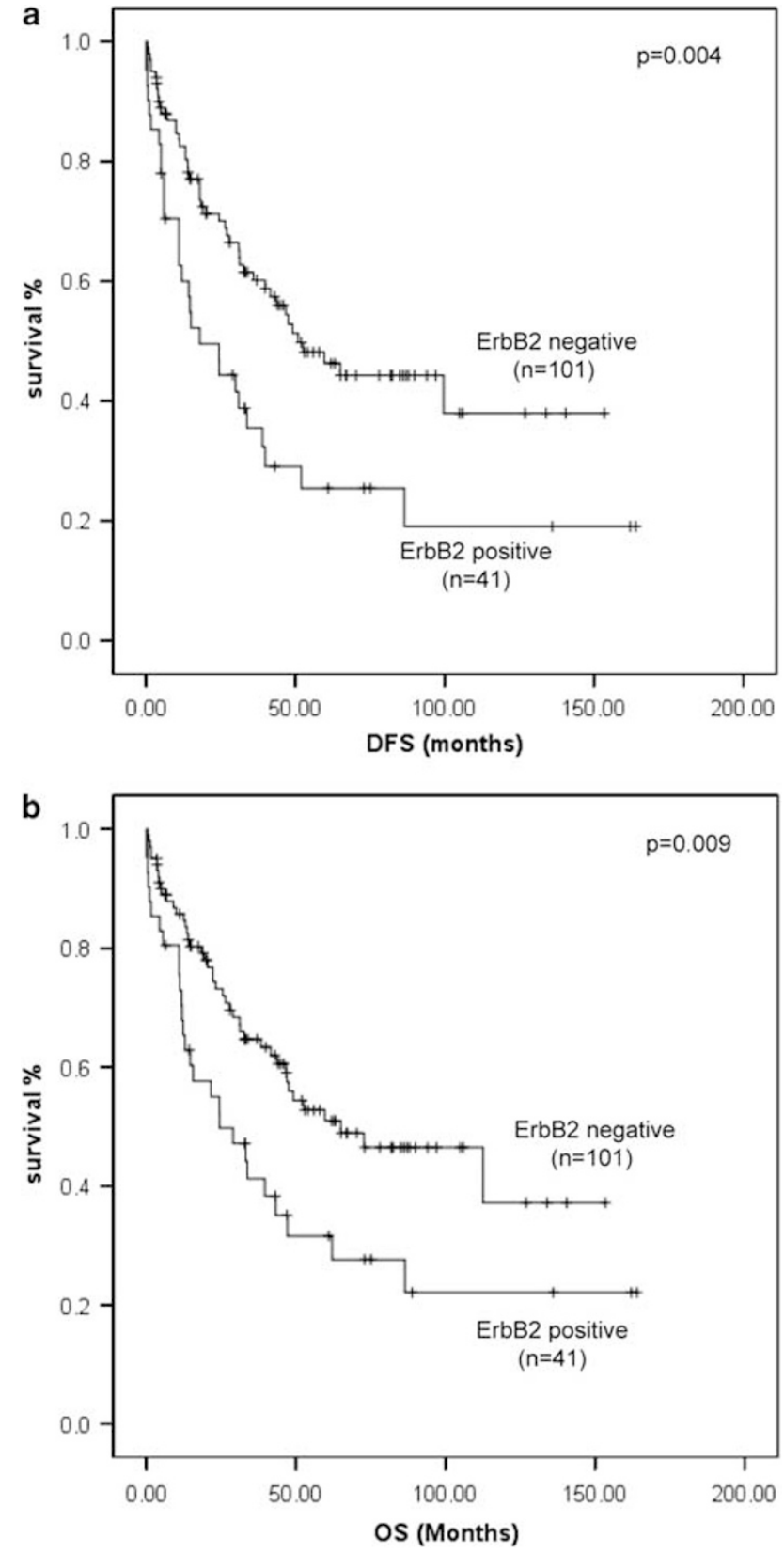

Figure 2 Survival analysis for ErbB2 in oesophageal adenocarcinomas. (a) ErbB2 and disease-free survival (DFS); (b) ErbB2 and overall survival (OS). ErbB2 positivity is defined as immunohistochemistry $3+$ and/or an ErbB2/Chr17 quotient of $>2$ as assessed by in situ hybridisation.

median overall survival was 73 months (95\% CI; 26-120 months). In contrast, the median diseasefree survival for patients with ErbB2-positive tumours was 18 months (95\% CI; 6-30 months), and the median overall survival was 25 months (95\% CI; 7-41 months). These differences were significant, both in univariate analysis $(P=0.004$ for disease-free survival and $P=0.002$ for overall survival, respectively) and in multivariate analysis $(P=0.019$ and 0.032 , respectively) that included the factors of pT category, presence of lymph node
Table 5 ErbB2 status and disease-free survival (a) and overall survival (b) (multivariate analysis)

\begin{tabular}{|c|c|c|c|c|}
\hline & \multirow[t]{2}{*}{$\operatorname{Exp}(B)$} & \multicolumn{2}{|c|}{$\begin{array}{l}95.0 \% \\
C I\end{array}$} & \multirow[t]{2}{*}{ Sign } \\
\hline & & Min & $\operatorname{Max}$ & \\
\hline \multicolumn{5}{|l|}{ (a) } \\
\hline pT-Category pT1-pT2-pT3/4 & 1.318 & 0.918 & 1.891 & 0.134 \\
\hline $\begin{array}{l}\text { Lymph node metastases } \\
\text { absent/present }\end{array}$ & 1.914 & 1.050 & 3.486 & 0.034 \\
\hline $\begin{array}{l}\text { Distant metastases } \\
\text { absent/present }\end{array}$ & 2.174 & 1.000 & 4.725 & 0.050 \\
\hline Grading G1-G2-G3/4 & 1.335 & 0.874 & 2.040 & 0.181 \\
\hline Resection-status R0-R1/2 & 2.556 & 1.329 & 4.916 & 0.005 \\
\hline ErbB2 (Her2) positive/negative & 1.771 & 1.098 & 2.856 & 0.019 \\
\hline \multicolumn{5}{|l|}{ (b) } \\
\hline pT-Category pT1-pT2-pT3/4 & 1.238 & 0.847 & 1.810 & 0.271 \\
\hline $\begin{array}{l}\text { Lymph node metastases } \\
\text { absent/present }\end{array}$ & 2.142 & 1.153 & 3.982 & 0.016 \\
\hline $\begin{array}{l}\text { Distant metastases } \\
\text { absent/present }\end{array}$ & 1.499 & 0.681 & 3.303 & 0.315 \\
\hline Grading G1-G2-G3/4 & 1.358 & 0.875 & 2.109 & 0.173 \\
\hline Resection-status R0-R1/2 & 3.123 & 1.608 & 6.064 & 0.001 \\
\hline ErbB2 (Her2) positive/negative & 1.709 & 1.046 & 2.792 & 0.032 \\
\hline
\end{tabular}

ErbB2 positivity is defined as immunohistochemistry $3+$ and/or an ErbB2/Chr17 quotient of $\geqslant 2$ as assessed by in situ hybridisation.

metastases, tumour differentiation, resection status and presence of distant metastasis at the time of surgery (Figure 2; Table 5).

\section{Discussion}

Previous studies examining ErbB2 (Her2) status in oesophageal adenocarcinomas observed a prevalence of ErbB2 protein overexpression or gene amplification in approximately $20-25 \%$ of cases with conflicting data about the prognostic significance of the amplification or overexpression. ${ }^{11,13,15}$ In this study, we tested ErbB2 status in oesophageal adenocarcinomas by in situ hybridisation (amplification) and immunohistochemistry (expression). A rate of ErbB2 positivity, which was defined $3+$ by immunohistochemistry and/or an ErbB2/Chr17 quotient of $\geq 2$ as assessed by in situ hybridisation, could be detected in $29 \%$ of the cases. Furthermore, ErbB2 positivity was associated with a worse clinical outcome in both the univariate and multivariate analyses.

The first aim of this study was to apply the modified ErbB2 immunoscoring method for gastric cancer, which was shown to be predictive of the response to trastuzumab-based therapies in patients with advanced gastric cancer in the ToGA study., ${ }^{3,8,10}$ For immunohistochemistry, we used the 4B5 assay. This antibody has also been approved by the FDA for ErbB2 testing in breast cancer and appeared to be at least as sensitive and may even show a higher inter-laboratory concordance for ErbB2 scoring and a closer relationship between immunohistochemi- 
cally $3+$ and ErbB2 gene amplification for gastric cancer. $^{10}$

There is the evidence that the determination of ErbB2 status by transferring the breast cancer immunohistochemistry scoring roles to gastric cancer may lead to a significant number of falsenegative ErbB2 tumours. ${ }^{20}$ Comparison between studies that have assessed ErbB2 in gastric cancer using the breast cancer evaluation system, on the one hand, and those that used the modified scoring system for gastric cancer, on the other hand, reveals significantly higher ErbB2 rates after the application of the proposed gastric cancer scoring system. Moreover, concordance between fluorescence in situ hybridisation and immunohistochemistry is better when using the gastric cancer scoring system; therefore, this evaluation system should be considered the scoring method of choice for immunohistochemical assessment of ErbB2 status in oesophagogastric adenocarcinomas. ${ }^{10}$ In a previous study, we also used the breast cancer scoring system (Dako; Hercept-test) for the assessment of ErbB2 in oesophageal adenocarcinomas and found a rate of $7 \% 2+$ cases and $10 \% 3+$ cases. $^{11}$ In this study, we demonstrated a significant correlation between the two scoring systems. However, there was an $18.1 \%$ discrepancy rate for the evaluation negative/ $1+v s 2$ $+/ 3+$.

For breast cancer and gastric cancer, in situ hybridisation is considered the standard method for the accurate determination of ErbB2 status in cases with equivocal immunohistochemical staining (ie, $2+$ ). ${ }^{9}$ In this study, we choose the novel, recently described bright field double in situ hybridisation for the assessment of ErbB2 amplification. This light-microscopy-based in situ hybridisation technique may be superior to conventional fluorescence in situ hybridisation for the investigation of small amplified tumour cell foci. ${ }^{18,21,22}$ This phenomenon frequently occurs in oesophagogastric carcinomas $^{1,3,23}$ and can readily be recognised by this method. We have compared bright field double in situ hybridisation data with fluorescence in situ hybridisation data from a previously published study ${ }^{12}$ and found a significant correlation between bright field double in situ hybridisation and both conventional fluorescence in situ hybridisation and the more complex but more sensitive 3D fluorescence in situ hybridisation. For breast cancer, similar observations were reported by others who described a high concordance in the assessment of ErbB2 amplification between fluorescence in situ hybridisation and the light-microscopy-based techniques of silver in situ hybridisation and bright field double in situ hybridisation, respectively, with advantages in terms of handling, orientation within the slides and in cases of intratumoral heterogeneity for bright field double in situ hybridisation. ${ }^{21}$

For the application of immunohistochemical staining and in situ hybridisation, we used tissue microarrays, which contained cores from a total of 142 oesophageal adenocarcinomas from which extensive clinico-pathological documentation was available. Unfortunately, we could not use one tissue microarray for the comparison of all methods because tissue microarrays constructed at two different time points were required. Nevertheless, the majority of cases were contained in both tissue microarrays so that correlation between immunohistochemistry, bright field double in situ hybridisation and fluorescence in situ hybridisation could be performed in approximately 100 cases. Discrepancies between the various in situ hybridisation methods and immunohistochemistry in our study may not only be caused by methodical or technical differences or the 'grey zone' of low-level gains ${ }^{12}$ but also represent the phenomenon of intratumoral heterogeneity of ErbB2 expression in gastro-oesophageal adenocarcinomas, ${ }^{23-26}$ because the cores of the donor blocks were obtained from slightly different tumour areas. Therefore, a heterogeneous distribution of ErbB2-positive cell clones may also be responsible for those divergent findings. This may be the case particularly for those cases with divergent results of high-level amplification. This phenomenon could also be observed in three cases within the same tissue microarray.

For the purpose of the determination of the prognostic impact of ErbB2 in oesophageal adenocarcinomas, this 'bias' of using two different tissue microarrays for the assessment of ErbB2 may therefore provide more reliable data about the ErbB2 status, because cores from different regions were investigated and false-negative results due to intratumoral heterogeneity may have been eliminated. Moreover, for the determination of ErbB2 status, if any amplification was observed on in situ hybridisation, including either fluorescence in situ hybridisation, 3D fluorescence in situ hybridisation or bright field double in situ hybridisation, the case was considered amplified based on the current recommendations for gastro-oesophageal carcinomas.

Compared to gastric cancer, comprehensive data about ErbB2 expression in oesophageal adenocarcinomas are relatively scarce due to the small sample sizes of homogenous collectives. In previous studies, we investigated ErbB2 expression in tissue microarrays of oesophageal adenocarcinomas using immunohistochemistry according to the breast cancer grading system and fluorescence in situ hybridisation (conventional and 3D fluorescence in situ hybridisation). ${ }^{11,12}$ We found a $7 \%$ rate of ErbB2 DAKO score $2+$ cases and a $10 \%$ rate of ErbB2 DAKO score $3+$ cases. $^{11}$ ErbB2 overexpression $(E r b B 2 / C H R 17 \geq 2)$ was demonstrated in $17 \%$ of cases by 3D fluorescence in situ hybridisation and in $13.1 \%$ of cases by conventional fluorescence in situ hybridisation, based on an analysis of at least 50 carcinoma cells per tissue core. Fluorescence in situ hybridisation data and immunohistochemistry data showed a significant correlation. However, a 
significant association between ErbB2 positivity and increased mortality could only be demonstrated by 3D fluorescence in situ hybridisation and not by immunohistochemistry or conventional fluorescence in situ hybridisation. Furthermore, a distinct subgroup of patients with a low-level gain of ErbB2 with unfavourable prognosis could be detected using 3D fluorescence in situ hybridisation. ${ }^{12}$ In a large study by a different group, the rate of ErbB2 overexpression/amplification was $15 \%$, and no prognostic impact with respect to patients' survival could be demonstrated. ${ }^{13}$ Others report similar rates of ErbB2 positivity in oesophageal adenocarcinomas, highlighting the impact of ErbB2 amplification and ERBB2 overexpression during the malignant progression of Barrett's metaplasia to invasive cancer. $^{27-29}$

The relatively high number of ErbB2-positive cases in our study compared with other reports may be due to the definition of ErbB2 positivity, which was based on the guidelines of the ToGA study and the EMEA for trastuzumab (Herceptin ${ }^{\odot}$ ) treatment for advanced gastric cancer. This recommendation requires a ErbB2 score of $3+$ by immunohistochemistry or an amplification level of $\geq 2$ (ErbB2/Chr17) even in small tumour cell clusters, ${ }^{10}$ which is different from the breast carcinoma requirements, in which cases with a ErbB2/ Chr17 ratio between 1.8 and 2.2 are considered as equivocal and ErbB2 expression is less heterogeneous. ${ }^{9}$ Furthermore, as mentioned above, falsenegative results may have been avoided by using various methods for the assessment of the amplification and intratumoral heterogeneity based on the investigation of tissue microarray cores from the same tumours from two different tissue miroarrays. On the basis of the results of our study, $\sim 30 \%$ of oesophageal adenocarcinoma patients would be potential candidates for ErbB2-targeted therapy. The original ToGA trial included metastatic gastric adenocarcinomas and adenocarcinomas of the gastric junction, ${ }^{8}$ which are described as distinct entities in the differentiation of oesophageal adenocarcinomas by the previous and most recent WHO classifications. ${ }^{30,31}$ However, the FDA and Health Canada approvals for trastuzumab (Herceptin ${ }^{\odot}$ ) treatment include all types of oesophagogastric adenocarcinomas; therefore, strict criteria for ErbB2 assessment in oesophageal adenocarcinomas are of the highest clinical relevance for selecting patients for ErbB2-targeted treatment, which may represent an expensive but promising additional therapeutic option for the advanced stages of this disease. ${ }^{32-36}$

In summary, in this study, we found that a significant number of oesophageal adenocarcinomas are positive for ErbB2. The assessment of ErbB2 by immunohistochemistry in combination with in situ hybridisation (bright field double in situ hybridisation or fluorescence in situ hybridisation) reveals highly relevant prognostic information and may also serve as a basis for targeted trastuzumab therapy.
Immunohistochemistry scoring as the primary method of ErbB2 assessment should be performed in accordance with the recommendations for gastric cancer. The method of evaluation of ErbB2 amplification by in situ hybridisation can be equivalently performed by conventional fluorescence in situ hybridisation or other light-microscopy-based methods, such as the novel bright field double in situ hybridisation technique.

\section{Acknowledgement}

We thank Mrs Petra Meyer and Mr Peter Strzelczyk for expert technical assistance.

\section{Disclosure/conflict of interest}

The authors declare no conflict of interest.

\section{References}

1 Grabsch H, Sivakumar S, Gray S, et al. HER2 expression in gastric cancer: rare, heterogeneous and of no prognostic value-conclusions from 924 cases of two independent series. Cell Oncol 2010;32:57-65.

2 Gravalos C, Jimeno A. HER2 in gastric cancer: a new prognostic factor and a novel therapeutic target. Ann Oncol 2008;19:1523-1529.

3 Hofmann M, Stoss O, Shi D, et al. Assessment of a HER2 scoring system for gastric cancer: results from a validation study. Histopathology 2008;52:797-805.

4 Marx AH, Tharun L, Muth J, et al. HER-2 amplification is highly homogenous in gastric cancer. Hum Pathol 2009;40:769-777.

5 Tanner M, Hollmen M, Junttila TT, et al. Amplification of HER-2 in gastric carcinoma: association with Topoisomerase IIalpha gene amplification, intestinal type, poor prognosis and sensitivity to trastuzumab. Ann Oncol 2005;16:273-278.

6 Allgayer H, Babic R, Gruetzner KU, et al. c-erbB-2 is of independent prognostic relevance in gastric cancer and is associated with the expression of tumorassociated protease systems. J Clin Oncol 2000; 18:2201-2209.

7 Park DI, Yun JW, Park JH, et al. HER-2/neu amplification is an independent prognostic factor in gastric cancer. Dig Dis Sci 2006;51:1371-1379.

8 Bang YJ, Van Cutsem E, Feyereislova A, et al. Trastuzumab in combination with chemotherapy versus chemotherapy alone for treatment of HER2-positive advanced gastric or gastro-oesophageal junction cancer (ToGA): a phase 3, open-label, randomised controlled trial. Lancet 2010;376:687-697.

9 Wolff AC, Hammond ME, Schwartz JN, et al. American Society of Clinical Oncology/College of American Pathologists guideline recommendations for human epidermal growth factor receptor 2 testing in breast cancer. J Clin Oncol 2007;25:118-145.

10 Ruschoff J, Dietel M, Baretton G, et al. HER2 diagnostics in gastric cancer-guideline validation and development of standardized immunohistochemical testing. Virchows Arch 2010;457:299-307. 
11 Langer R, Von Rahden BH, Nahrig J, et al. Prognostic significance of expression patterns of c-erbB-2, p53, p16INK4A, p27KIP1, cyclin D1 and epidermal growth factor receptor in oesophageal adenocarcinoma: a tissue microarray study. J Clin Pathol 2006;59: 631-634.

12 Rauser S, Weis R, Braselmann H, et al. Significance of HER2 low-level copy gain in Barrett's cancer: implications for fluorescence in situ hybridization testing in tissues. Clin Cancer Res 2007;13:5115-5123.

13 Reichelt U, Duesedau P, Tsourlakis M, et al. Frequent homogeneous HER-2 amplification in primary and metastatic adenocarcinoma of the esophagus. Mod Pathol 2007;20:120-129.

14 Tapia C, Glatz K, Novotny H, et al. Close association between HER-2 amplification and overexpression in human tumors of non-breast origin. Mod Pathol 2007;20:192-198.

15 Walch A, Bink K, Hutzler P, et al. HER-2/neu gene amplification by FISH predicts poor survival in Barrett's esophagus-associated adenocarcinoma. Hum Pathol 2000;31:1332-1334.

16 Cunningham D, Allum WH, Stenning SP, et al. Perioperative chemotherapy versus surgery alone for resectable gastroesophageal cancer. $\mathrm{N}$ Engl J Med 2006;355:11-20.

17 Langer R, Ott K, Feith M, et al. Prognostic significance of histopathological tumor regression after neoadjuvant chemotherapy in esophageal adenocarcinomas. Mod Pathol 2009;22:1555-1563.

18 Nitta H, Hauss-Wegrzyniak B, Lehrkamp M, et al. Development of automated brightfield double in situ hybridization (BDISH) application for HER2 gene and chromosome 17 centromere (CEN 17) for breast carcinomas and an assay performance comparison to manual dual color HER2 fluorescence in situ hybridization (FISH). Diagn Pathol 2008;3:41.

19 Sobin L, Gospodarowicz ML, Wittekind Ch. TNM Classification of Malignant Tumors. John Wiley \& Sons: New York, 2010:66-71.

20 Holmes JL, Sharp SY, Hobbs S, et al. Silencing of HSP90 cochaperone AHA1 expression decreases client protein activation and increases cellular sensitivity to the HSP90 inhibitor 17-allylamino-17-demethoxygeldanamycin. Cancer Res 2008;68:1188-1197.

21 Dietel M, Ellis IO, Hofler $\mathrm{H}$, et al. Comparison of automated silver enhanced in situ hybridisation (SISH) and fluorescence in situ hybridisation (FISH) for the validation of HER2 gene status in breast carcinoma according to the guidelines of the American Society of Clinical Oncology and the College of American Pathologists. Virchows Arch 2007;451:19-25.

22 Fritzsche FR, Bode PK, Moch H, et al. Determination of the Her-2/neu gene amplification status in cytologic breast cancer specimens using automated silver-enhanced in-situ hybridization (SISH). Am J Surg Pathol 2010;34:1180-1185.

23 Walch A, Bink K, Gais P, et al. Evaluation of c-erbB-2 overexpression and Her-2/neu gene copy number heterogeneity in Barrett's adenocarcinoma. Anal Cell Pathol 2000;20:25-32.

24 Geddert H, Zeriouh M, Wolter M, et al. Gene amplification and protein overexpression of c-erb-b2 in Barrett carcinoma and its precursor lesions. Am J Clin Pathol 2002;118:60-66.

25 Langer R, Specht K, Becker K, et al. Association of pretherapeutic expression of chemotherapy-related genes with response to neoadjuvant chemotherapy in Barrett carcinoma. Clin Cancer Res 2005;11: 7462-7469.

26 Owonikoko T, Rees M, Gabbert HE, et al. Intratumoral genetic heterogeneity in Barrett adenocarcinoma. Am J Clin Pathol 2002;117:558-566.

27 Fritcher EG, Brankley SM, Kipp BR, et al. A comparison of conventional cytology, DNA ploidy analysis, and fluorescence in situ hybridization for the detection of dysplasia and adenocarcinoma in patients with Barrett's esophagus. Hum Pathol 2008;39: 1128-1135.

28 Walch A, Specht K, Bink K, et al. Her-2/neu gene amplification, elevated mRNA expression, and protein overexpression in the metaplasia-dysplasia-adenocarcinoma sequence of Barrett's esophagus. Lab Invest 2001;81:791-801.

29 Walch A, Specht K, Braselmann H, et al. Coamplification and coexpression of GRB7 and ERBB2 is found in high grade intraepithelial neoplasia and in invasive Barrett's carcinoma. Int J Cancer 2004;112:747-753.

30 Bosman FT, Carneiro F, Hruban RH, et al. (eds). WHO Classification of Tumours of the Digestive System, 4th edn. World Health Organization: Lyon, 2010, pp 40.

31 Hamilton S, Aaltonen LA, (eds). Classification of Tumours. Pathology and Genetics of Tumours of the Digestive System, 3rd edn. World Health Organisation IARC Press: Lyon, 2000, pp 31.

32 Bekaii-Saab TS, Roda JM, Guenterberg KD, et al. A phase I trial of paclitaxel and trastuzumab in combination with interleukin-12 in patients with HER2/neu-expressing malignancies. Mol Cancer Ther 2009;8:2983-2991.

33 Homs MY, Voest EE, Siersema PD. Emerging drugs for esophageal cancer. Expert Opin Emerg Drugs 2009;14:329-339.

34 Safran H, Dipetrillo T, Akerman P, et al. Phase I/II study of trastuzumab, paclitaxel, cisplatin and radiation for locally advanced, HER2 overexpressing, esophageal adenocarcinoma. Int J Radiat Oncol Biol Phys 2007;67:405-409.

35 Safran H, DiPetrillo T, Nadeem A, et al. Trastuzumab, paclitaxel, cisplatin, and radiation for adenocarcinoma of the esophagus: a phase I study. Cancer Invest 2004;22:670-677.

36 Villanacci V, Rossi E, Grisanti S, et al. Targeted therapy with trastuzumab in dysplasia and adenocarcinoma arising in Barrett's esophagus: a translational approach. Minerva Gastroenterol Dietol 2008;54: 347-353. 